\title{
Energy Efficiency in Heterogeneous Wireless Access Networks
}

\author{
Shobanraj Navaratnarajah, Arsalan Saeed, Mehrdad Dianati and Muhammad Ali Imran \\ Centre for Communication Systems Research (CCSR) \\ University of Surrey, Guildford, Surrey, GU2 7XH, UK. \\ Email: \{s.navaratnarajah, arsalan.saeed, m.dianati,m.imran\}@surrey.ac.uk
}

\begin{abstract}
In this paper, we bring forward the important aspect of energy savings in wireless access networks. We specifically focus on the energy saving opportunities in the recently evolving heterogeneous networks (HetNets), both Single-RAT and MultiRAT. Issues such as sleep/wakeup cycles and interference management are discussed for co-channel Single-RAT HetNets. In addition to that, a simulation based study for LTE macro-femto HetNets is presented, indicating the need for dynamic energy efficient resource management schemes. Multi-RAT HetNets also come with challenges such as network integration, combined resource management and network selection. Along with a discussion on these challenges, we also investigate the performance of the conventional WLAN-first network selection mechanism in terms of energy efficiency (EE) and suggest that $\mathrm{EE}$ can be improved by the application of intelligent call admission control policies.
\end{abstract}

Index Terms-Energy efficiency, Heterogeneous Networks, Long-Term Evolution, Multi-RAT, Femtocell, WLAN.

\section{INTRODUCTION}

$\mathbf{T}$ HE evolution of wireless communication devices continues to explode the traffic demand in wireless communication systems. It is expected that the traffic demand will increase up to thirteen fold by 2017 as that of 2012, at a compound annual growth rate (CAGR) of $66 \%$ [1]. Therefore, wireless network providers face an enormous challenge to increase their network capacity, in order to cope with the increasing traffic demand. Since improvements in spectral efficiency (SE) at link level approaches its theoretical limits with currently existing technologies, the next generation of technology is about improving spectral efficiency per unit area [2]. Therefore, network providers and equipment vendors are looking into an evolved network topology to improve the network capacity. To this end, the heterogeneous network (HetNet) architecture is seen as a promising solution to the capacity problem of wireless communication networks. A HetNet may consist of different size of cells with different radio access technologies (RATs). Fig. 1 depicts a typical example of a HetNet. In HetNets, small cells bring down the distance between transmitter and receiver, which results in low pathloss. This leads to an increased received signal power, signal to noise ratio (SNR) and better SE. Therefore, the area efficiency (AE) (i.e SE per unit area) can be improved [3].

Apart from the capacity demand, energy consumption of mobile terminals becomes an increasing concern due to increased network usage of latest advanced wireless communication devices (e.g. smart phones and tablet PCs). Therefore,

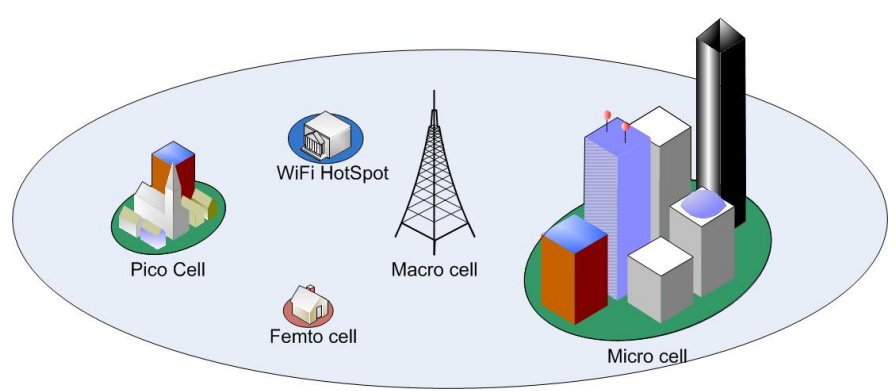

Fig. 1: A typical example of HetNet

there is a significant threat that the $4 \mathrm{G}$ mobile users will be searching for power outlets rather than network access, and once again binding them to a single location. This problem is sometimes described as the energy-trap of $4 \mathrm{G}$ systems [4]. At the same time, EE of the network also considered as an important aspect of network operation, due to the increased cost of energy and environmental concerns. Hence, apart from the coverage, capacity and QoS, the energy efficiency (EE) also becomes an important performance indicator from the component design to the network operation.

Studies show that major part of the energy (50-80\%) is consumed in the wireless access part of the mobile communication networks [5]. Hence, improvement in EE of access networks, will have considerable impact on the total network EE. In this regard, the HetNet architecture can also be considered as a promising solution to improve the network EE. In HetNets, particularly in small cells, the required transmit power is very low compared to macro cells, due to the short distance between transmitter and receiver [3]. This enables the base station (BS) to be made with less complexity and improved power efficiency. For example, such low power small BS does not require any cooling systems. Further, the reduction in transmit power requirement, can improve the battery life of mobile devices. Traditionally, there is a trade-off between SE and EE at link level. However, with the HetNet architecture, it is possible to improve EE of the network while improving AE. Therefore, HetNets attract considerable interest from mobile communication industry. Particularly, deployment of femtocells and WiFi hot spots attract significant interest, due to the fact that, apart from the aforementioned advantages, they can considerably reduce the deployment and operational costs. It is estimated that $33 \%$ of mobile data traffic was off-loaded to 
fixed network through WiFi and femto-cells in 2012, and it is expected to reach $46 \%$ in 2017 [1].

In this article, we investigate the challenges and opportunities to improve EE in HetNets. Particularly, in LTE-WiFi and LTE macro-femto HetNets as Multi-RAT and Single-RAT HetNet, respectively. The article is organised as follows. In the following section, we discuss the deployment aspects of HetNets. Then, we discuss the major challenges in MultiRAT HetNets, such as network integration, combined resource management, network selection and vertical handover. Further, we investigate the EE performance of mainstream network selection scheme for cellular-WLAN network, known as $W L A N$-first, through system level simulations that consider all practical aspects of LTE-WLAN HetNet. We show that, with appropriate resource management policy, total network EE of Multi-RAT HetNets can be significantly improved. Then, we discuss the areas with energy saving potentials in SingleRAT HetNets, such as sleep modes and interference mitigation techniques. We also identify with a simulation based study for LTE macro-femto HetNets, that the interference amongst the transmission nodes severely deteriorates the EE of the network. Further, we also discuss different frequency reuse schemes and their effects on EE for a LTE macro-femto HetNet. We suggest that there is need for development of dynamic resource allocation algorithms to improve the EE as well as capacity of such Single-RAT HetNets. Finally, we conclude the article.

\section{HetNet Deployment}

In terms of network deployment, a HetNet can consist of different size of cells, such as macro, micro, pico and femto cells that provide services to same coverage area in a multi-tier configuration, that utilise single RAT. This kind of HetNet is known as Single-RAT HetNet. For example, the 3rd Generation Partnership Project (3GPP) Long Term Evolution Advanced (LTE-A) system, with outdoor macro Base Station (BS) and indoor Home BS (HBS) is a prime example for such Single-RAT HetNet. On the other hand, in a Multi-RAT HetNet, multiple RATs such as Wideband Code Division Multiple Access (WCDMA), Worldwide Interoperability for Microwave Access (WiMAX), Wireless Local Area Network (WLAN) and LTE can jointly provide service to same coverage area in a complementary manner with different coverage ranges. A network of outdoor WCDMA/LTE macro cells with indoor and hot spot coverage of WLAN is a practical example of Multi-RAT HetNets.

The advantage of Single-RAT HetNets comes form the relatively less complex network operation compared to the MultiRAT. For example, a Multi-RAT HetNet needs additional authentication, authorization and accounting (AAA) system, allowing users to perform authentication and authorization processes in different RATs, attending to security suites and subscription profiles for security and billing purposes. Where as in Single-RAT HetNet such additional mechanism is not required. However, Single-RAT HetNet suffers form the crosstier interference. Since the spectrum is scare and expensive, the available licensed spectrum is limited to each operator.
Therefore, in most cases, the same spectrum will be shared between different tiers in a Single-RAT HetNet. To this end, mitigating interference while increase the network capacity, is considered as a major challenge in Single-RAT HetNet. On the other hand, in a multi-RAT HetNet, the advantage is, having different RATs that utilise different frequency spectrum including the unlicensed spectrum (e.g. WiFi). Therefore, Multi-RAT HetNet does not suffer from cross-tier interference. However, integration of different RATs becomes one of the major problem in multi-RAT HetNet, due to different technological and architectural aspects of each RAT.

\section{Multi-RAT HetNet}

Due to the technological improvements and innovations, various kinds of wireless access technologies were developed and deployed over the past decades. During the initial development of each RAT, the available technology, design objectives, requirements, and regulations lead to different access technologies. For example, traditional cellular (e.g. GSM) technologies developed primarily for voice service with the objective of providing large coverage and service continuity on the move. Whereas WLAN technologies developed for providing high bandwidth, short range, low cost solution for data services. To this reason, at present, there are many wireless access technologies available to serve the user depends on the the users service requirements. However, each RAT has its own benefits and limitations based on the service it can provide to the user (e.g. coverage, bandwidth and monetary cost, etc). Therefore, one of the main objectives of $4 \mathrm{G}$ (also sometimes known as beyond $3 \mathrm{G}$ (B3G) systems) was to integrate all these different access technologies into a common network, sometime referred as Open Wireless Architecture (OWA), that provides Always Best Connected (ABC) services to the users, where the users able to choose the best available access networks in a way that best suits their needs [6]. Therefore, MultiRAT HetNet has been identified as the future architecture of the wireless communication systems. However, there are many challenges related to the Multi-RAT heterogeneous networks, such as network integration and resource management, network selection and handover, security, etc.

\section{A. Network Integration}

Since, each RAT has developed with different architecture and protocols, integration of different RATs has been a major challenge in a Multi-RAT environment. Especially access technologies developed based on 3GPP and IEEE 802.x specifications are developed independently at the beginning, without considering any cooperation between these two systems. However, in order to efficiently operate the Multi-RAT HetNet, integration of different RATs is essential. To this end, recently, there has been a considerable effort to address this problem from the research community and standardisation bodies. For example, one of the objectives of European Ambient Networks project [7] was to enable the cooperation of heterogeneous networks belonging to different operator and/or technology domains. With respect to standardization, the IEEE standard 802.21, called Media Independent Handover (MIH) Services, 
defines extensible media access independent mechanisms that enable the optimization of handovers between heterogeneous IEEE 802.x systems, and facilitate handovers between IEEE 802.x systems and cellular systems [8]. Further, the 3GPP specifications TS 23.234 specifies the system description for interworking between 3GPP systems and Wireless Local Area Networks (WLANs), while TS 23.327 specifies the system description for providing mobility between 3GPP Wireless Local Area Network Interworking (I-WLAN) and 3GPP Systems. In addition, TS 23.327 also defines a technical solution with necessary enhancement of the I-WLAN architecture to support mobility and roaming between 3GPP-WLAN Interworking system and 3GPP Systems.

\section{B. Resource management}

Due to the complex architecture of Multi-RAT networks that involve different access protocols, network resource management becomes another major challenge. Resource management techniques such as radio resource management (RRM) and call admission control (CAC) are known as Joint RRM (JRRM) or Common RRM (CRRM) and Joint CAC (JCAC) in a MultiRAT environment. It is evident that the adopted resource management strategy can have considerable effect on the overall network performance. There are many CRRM and JCAC solutions proposed in the literature to optimise the network operation in Multi-RAT HetNet. However, most of the existing solutions focus on improving system performance in terms of throughput, network load balancing, user experience, call dropping and blocking probabilities, and battery life of user terminal. There is a lack of studies that investigate, and provide solutions to improve the EE of the whole network of a Multi-RAT HetNet. Since the total network EE becomes an important performance indicator, it is worth to investigate the resource management strategies in order to improve the $\mathrm{EE}$ of the whole network.

\section{Network Selection and Vertical Handover}

Depending on whether the user initiates a session or moves during an active session across different RATs, the operation of choosing a desired network is known as network selection or vertical handover decision (VHD), respectively. Making optimal network selection or VHD, in Multi-RAT HetNet is another challenging problem. There are some advanced network selection or VHD scheme in literature [9]. The adopted network selection or VHD scheme can have considerable effect on the overall network performance. So far, the objectives of available network selection or VHD schemes in the literature, have been mainly limited to throughput enhancement, enabling seamless mobility, and load balancing. When it comes to EE, only battery life extension techniques of mobile terminals have attracted some attention due to their limited source of energy. Therefore, optimizing total network EE through advanced network selection or VHD solution will be an interesting research area.

\section{EE Analysis of LTE-WiFi HetNet}

Traditionally, total network EE has not been an optimization parameter in a Multi-RAT HetNet. Moreover, in current
cellular-WLAN HetNets, the user terminals select the desired network based on the user preference, without specific optimisation, due to the complexity involved in such optimisation processes. For example, in the widely used network selection scheme, known as WLAN-first [10], the mobile terminals always connect to the available WLAN, without considering network load, quality of service (QoS) or EE. Further, there is no CAC policy in the WLAN-first scheme. Therefore, the WLAN network can become congested, hence the whole network performance degrades. To this end, we investigate the performance of LTE-WiFi HetNet in terms of total network EE and per user throughput for WLAN-first scheme with and without CAC. Here, when there is a CAC policy applied to the WiFi network, we assume that the APs only allow certain number of users (e.g. 4 users) who have best channel condition under its coverage.

For this study, we consider an LTE-WiFi HetNet comprising of a single LTE cellular macro base station (BS) and multiple WiFi access points (APs), providing service to the same coverage area. Fig. 2 depicts such typical network architecture. Since we are interested in access part of the network, we adopt tight coupled network of LTE and WiFi, where the WiFi APs are connected to the Evolved Packet Core (EPC) through a gateway router in a same manner as the LTE BS (eNodeB). We evaluate the system performance through Network Simulator 3 (NS3) based system level simulations, adopting realistic power consumption models for both networks and considering all practical aspects of full communication protocol stack according to the relevant standards. For energy consumption evaluations, we adopted power consumption profile for macro BS and WiFi AP from [11] and [12] respectively. Fig. 3 and 4 show the simulation results in terms of per user throughput and normalised EE respectively, with respect to increased AP deployment in the considered coverage area.

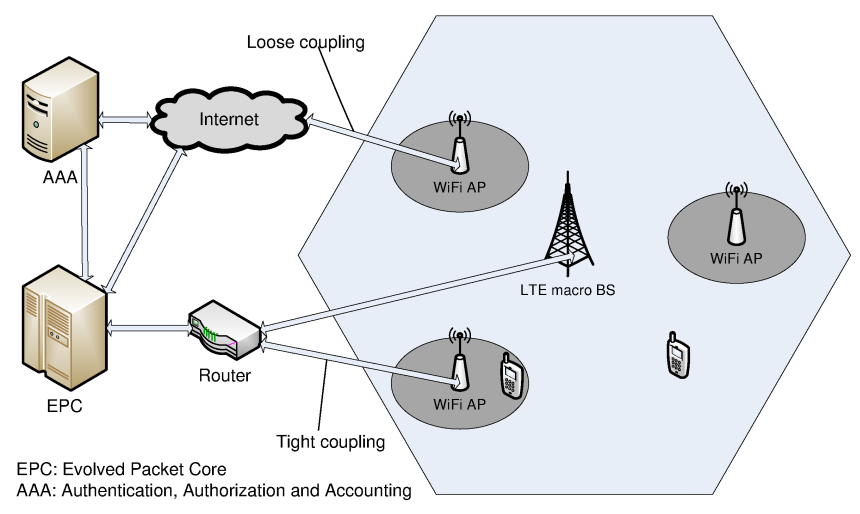

Fig. 2: Typical LTE-WiFi HetNet

From Fig. 3, we can see that, when WiFi APs are introduced in the LTE macro coverage area at a low number, the system performance in terms of average user throughput has a slight improvement. This is due to the fact that, the sparse nature of APs deployment, helps the system to off-load some users to WiFi from the LTE macrocell, without interfering with each other. However, when the AP penetration increases, average user throughput degrades severely. This is due to the increased 
interference (i.e. collision of frames) in WiFi system. Since without CAC, large number of users can select WiFi, the higher number of contenting and hidden nodes degrade the system performance. However, by applying a CAC policy that limits the number of users can be served by an AP as well as only selecting the best users in terms of channel condition under WiFi coverage, the per user throughput can be significantly improved.

From Fig. 4, we can see the same effect of applying an intelligent CAC in WiFi system in terms of EE. As we can see in the simulation results, by applying some intelligent CAC policy, the whole system performance in terms of average user throughput and total network EE can be significantly improved. Therefore, it is not beneficial to just off-load the traffic in a Multi-RAT HetNet without optimising the network through advanced operation policies. For example, the mainstream WLAN-first scheme without any CAC policy is not beneficial in terms of throughput or EE.

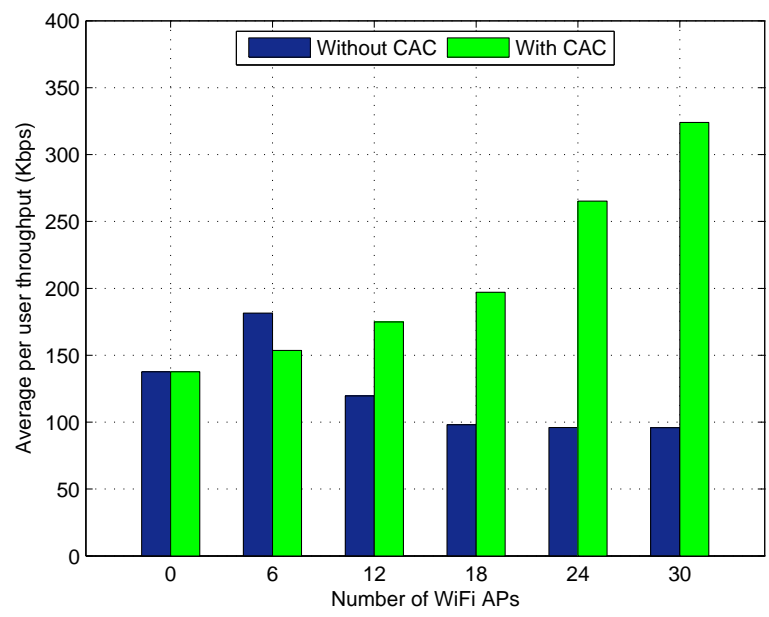

Fig. 3: Average per user throughput in a LTE-WLAN HetNet with and without an intelligent CAC policy.

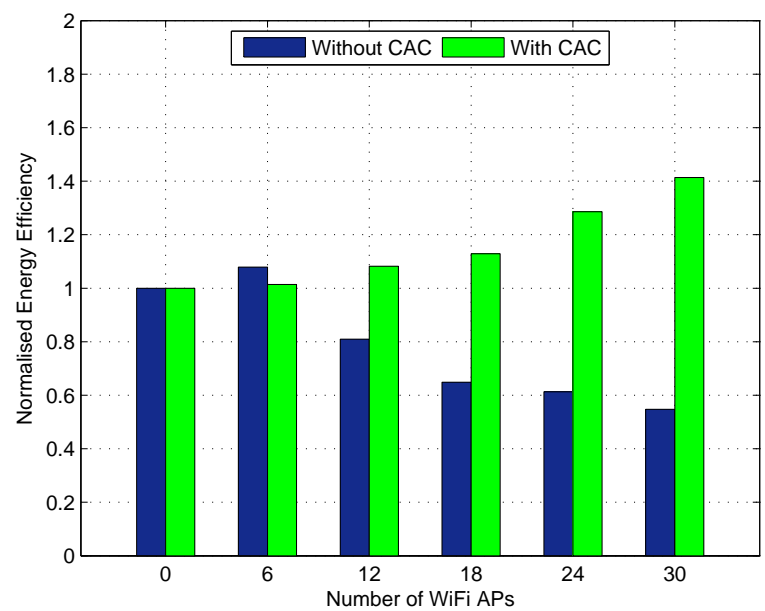

Fig. 4: Total network energy efficiency in a LTE-WLAN HetNet with and without an intelligent CAC policy.

\section{Single-RAT HetNet}

In the early days of mobile communications, macro cells with a larger coverage area were deployed by operators. As the capacity requirements increased, various advancements such as use of wider spectrum, slicing and reusing the spectrum and better modulation schemes were proposed. Despite that, reducing the cell radius is considered to provide the highest capacity gains. Today the use of micro and pico cells is also not sufficient enough to fulfil the data requirements of users in hotspots and indoors. Femtocells are the way forward to address these high data demands. However, the deployment of femtocells comes with certain challenges. Even though, the femtocells have low power consumption profile, one of the major concern is the high aggregated energy consumption, due to high deployment rate and underutilisation.

\section{A. Small Cells Access types}

Operator deployed Femto Access Points (FAPs) are usually known as Open Subscriber Group (OSG) FAPs, which offload the macro cell traffic and serve cell edge users. Another category of FAPs is known as Closed Subscriber Group (CSG) FAPs, these as prominent from the name, are closed to all the users except for certain high priority users. Such CSG FAPs are usually deployed by subscribers and only open to a limited set of users. Co-Channel operation of CSG FAPs poses severe interference to non-CSG users who remain within the vicinity of these CSG FAPs. Another much flexible access type of FAPs are known as hybrid access group, such hybrid FAPs act as CSG FAPs except that in presence of a non-CSG users, certain basic resources are shared to minimise interference to non-CSG users.

\section{B. Optimal deployment of small cells}

Deployment of small cells, in hotspots and cell edges is beneficial from QoS as well as energy efficiency point of view. But these gains come at the cost of deploying FAPs at ideal locations, either where data requirements are high or macrocell performance is low. However, it is very difficult to predict the optimal locations of these nodes. Considering the dense subscriber owned deployment of FAPs, they may not be beneficial in terms of EE, since these small cells are operational all the time. Even if there are no users to be served, a substantial amount of circuit energy is being drawn by these nodes. Dense deployment of FAPs can be energy efficient if the full potential of the capacity gains are utilised. Considering the expected heavy deployment of FAPs in near future and the dynamic traffic demands, sleep modes are the most potential solution to overcome the wastage of energy in case of low traffic demands.

\section{Sleep Modes in Small Cells}

A transmission node can be in a number of states, along with load conditions. Load on the node mainly effects the total transmission energy consumption, where as the states of the node determine its circuit energy consumption. Other than the active (i.e. full operational) state, a macro/femto node can 
be in idle or sleep mode, where there is no user activity or most of the modules are turned off respectively. Since, indoor home and enterprise FAPs are not utilised most of the time, the idle state energy gets wasted. Switching the node to sleep mode significantly reduces the energy consumption.

The simplest sleep mode technique is where almost all the modules of a BS are shut down based on a fixed timer. This timer is manually configured for a statistical traffic cycle, usually during few hours of night when user traffic is very low. A drawback of such a scheme is very obvious that since the sleep mode cycle is static and only based on traffic statistics, in event of unusual activity the system performance might degrade or needs to be manually reconfigured. In case of small home base stations such conditions shall arise on frequent basis and is not feasible to reconfigure. Adaptive sleep modes based on dynamic traffic monitoring are the way forward. Dynamic sleep/wakeup function of the node can be categorised into three types; node controlled, UE controlled and core network controlled [13].

1) Node controlled mode: In this mode, the FAP does pilot sensing for call activity, while the UE is attached to a underlay macro node. As the UE activity is sensed, the FAP becomes active and the UE gets served by the FAP with better QoS. For this self controlled operation, a micro controller and a sniffer is added to the FAP, to control the sleep/wakeup cycle. One drawback of such a technique could be the unnecessary wakeup of CSG FAPs in presence of activity from a non-CSG UE.

2) UE controlled mode: In this mode, the UE periodically sends wakeup messages to FAPs. Such a scheme can be very energy efficient for FAPs, as a FAP only listens for wakeup messages. But this might increase energy consumption at the UE's end due to excessive wakeup message when there is no FAP in the vicinity.

3) Network controlled mode: In this mode, the core network sends sleep/wakeup message to the FAP over the backhual link which can be a S1 or a logical X2-Interface. As evident from the explanation that this scheme requires a control interface between the FAP the the core network, increasing the load on the network as well as the FAP needs to maintain an active backhaul link. There are also concerns regarding the state control of FAPs over the DSL/internet backhaul connections, where a dedicated backhaul link is not available. Keeping these categories of sleep modes in consideration and the geographic nature of FAPs, there is a need for development of detailed sleep mode procedures with maximum autonomous capabilities built-in within the FAPs.

\section{Inter-Cell Interference Management}

Interference amongst the transmission nodes causes severe spectral as well as energy inefficiency. Interference can be mitigated by interference cancellation, interference randomization or interference avoidance techniques. However, interference avoidance schemes tend to be less complex and more efficient in cellular networks [14].

1) Frequency Reuse Schemes: Frequency reuse based techniques were proposed for homogeneous networks usually macro/micro cells. The basic concept was the reuse of the available spectrum in a way to maximise the spectrum usage while minimising the interference among the neighbour cells. The most simplest form of reuse schemes is Reuse 1 scheme, where the whole available spectrum is used by all the cells in a network hence maximising the spectrum usage. However, such a scheme introduces severe interference among the neighbour cells. The other simple reuse scheme is Reuse 3, where the available spectrum is divided into three portions and different portions of spectrum are allocated to adjacent cells. Such a scheme reduces the interference amongst the neighbouring nodes while reducing the spectrum usage. Fractional frequency reuse is another more advanced frequency reuse technique, where each cell is divided into major and minor regions, usually central and edge regions. There are various variant of fractional frequency reuse in literature but the most prominent concept is the maximum spectrum usage for the central region and low spectrum usage in the cell edge region to minimise interference.

2) Cell Coordination Based Schemes: To overcome the static behaviour of the above mentioned frequency allocation schemes and make the network adaptive to traffic requirements, coordination amongst the transmission sites was discussed as a possible solution. Cell coordination can be at different levels within the network. A central entity depending on the level of coordination makes decisions for the allocation of resources, to minimise the overall interference in the network. In a centralised cell coordination based scheme, all the BSs report various information to the central identity within the network, this entity responds back with resource allocation information. Such a scheme is theoretically ideal but very difficult to implement in a real system, due to the heavy exchange of information and delays with in the network. A semi-distributed coordination is neither completely central nor distributed. Rather it is form of coordination amongst a group of cell sites, governed by a central entity for the specific coordination group.

Distributed Coordination based schemes are also known as de-centralised schemes. The prime difference from the above mentioned scheme is that there is no central entity required. The cell sites coordinate and optimise their resources, possibly following a network policy. This scheme is further categorised into Coordinated-Distributed scheme and Autonomous-Distributed scheme. As obvious from the name, Coordinated-Distributed scheme is where the cell sites interact with each other for the exchange of information and optimise there performance and resources, based on a selfish per node algorithm. In an Autonomous-Distributed scheme, there is no exchange of information amongst the nodes, hence completely eliminating the coordination load due to information exchange. Each node self organises itself based on the network policies or in a more advanced scheme, based on sensing nearby interference and users.

3) Enhanced Inter-Cell Interference Coordination: Since the previously mentioned frequency reuse based schemes were more appropriate for macrocells having a rather manageable geographic coverage area, in case of small cells such as FAPs these scheme are not appropriate. To address these concerns 
more advanced schemes are discussed in 3GPP specifications, known as Enhanced Inter-Cell Interference Coordination (eICIC) schemes. The three broader categories of eICIC techniques are time domain techniques, frequency domain techniques and power control.

Time domain techniques are focused on mitigating interference to victim UE from a dominant interferer by restricting time domain resources. These techniques are proved to be very efficient in reducing interference, however need strict time synchronization amongst the transmission nodes.

Power control techniques are one of the most widely discussed techniques in the 3GPP community. The basic concept is the reduction in the transmit power of the interfere to reduce the interfere to the nearby victim UE.

Frequency domain techniques are also known as reduced bandwidth schemes, these are very similar to the previously discussed ICIC techniques, but consider HetNet operation and interference avoidance for data channels as well as control channels. In presence of a victim UE, the FAP restricts its transmissions in certain portions of available bandwidth.

To this end, we can conclude that there are a number of interference mitigation techniques available in literature, however these are more suitable for larger cell sites such as macro and micro cells. For smaller cells, eICIC techniques discussed in specifications are appropriate but need further development of dynamic and autonomous resource management techniques.

\section{E. EE Analysis of LTE macro-femto HetNet}

Similar to our previous EE analysis for LTE-WiFi HetNets, we simulate a LTE macro-femto HetNet with a single LTE macro BS and several uniformly random distributed FAPs and UEs. In order to analyse the energy consumption of the network, we transfer a fixed size file to each user from a remote host. We adopt downlink power consumption profiles for macro and femtocells from [11]. To make our assumptions realistic, a maximum of four users are attached to each FAP. We simulate four cases to investigate the effects of interference on the energy consumption of the network. Ideal case is where there is no interference amongst the node, or each node transmits in a dedicated band. Though this case is not practical for an operator to implement, but serves as a benchmark in our analysis. Case $A$ is where there is a Reuse 1 in the network, and every transmission node interferes with the neighbouring node. In Case B, the macro node and FAPs operate in separate frequency bands, therefore, FAPs do not interfere with the macro node and vice versa. However, there is a Reuse 1 amongst the FAPs, so FAPs interfere with each other. In Case $C$, we simulate a static partitioning of the bandwidth where the available $20 \mathrm{MHz}$ of bandwidth is divided amongst macro node $(15 \mathrm{MHz})$ and FAPs $(5 \mathrm{MHz})$, so the macro node operated in a reduced dedicated bandwidth and there is interference amongst the FAPs only.

The results in Fig. 5 show that for the Ideal Case, the energy consumption decreases as we increase the density of FAPs. So increment in density of FAPs is beneficial if the FAPs do not cause interference within the network. In case of Case A, the energy consumption is higher compared to the Ideal case, due to the interference among the nodes. In Case B, since there is no interference between macro and femto cells, the energy consumption of the network is nearly same as that of Ideal case, up to certain number of FAPs. However, at higher density of FAPs, energy consumption increases due to the interference amongst the FAPs. In Case C, we can observe that the static partitioning of the spectrum is not at all energy efficient compared to all other cases.

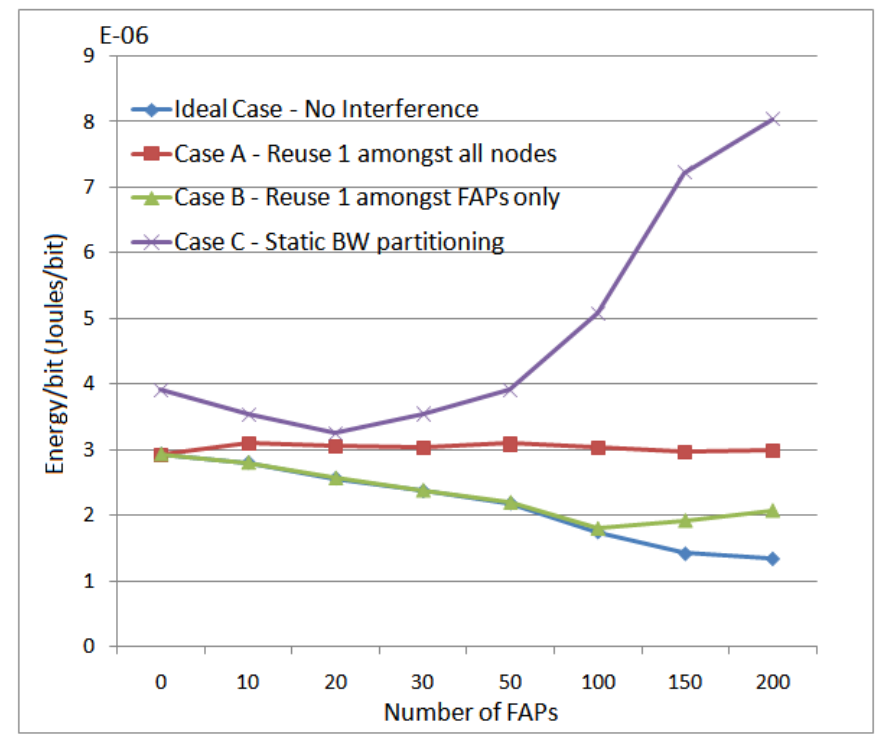

Fig. 5: Energy/bit comparison for Case A, B and C (50 Users)

\section{Conclusions}

Due to the recent evolution of mobile communication devices, demand for network capacity increases exponentially. At the same time, the energy efficiency (EE) of both wireless communication devices and network attracts increasing interests due to short battery life time of advanced mobile terminals and increasing operation cost of mobile networks. The HetNet architecture is considered as a promising solution for both aforementioned capacity and EE problems. Therefore, in this article, we summarise the challenges and opportunities to improve the EE while increasing the network capacity in both Multi-RAT and Single-RAT HetNet. Especially, in LTEWiFi and LTE macro-femto HetNet respectively. It is evident that, through proper network operation policies and resource management strategies, the total network EE can be improved while increasing the network capacity by off-loading the traffic to WiFi hot spots or femto cells.

\section{ACKNOWLEDGMENT}

This work has been done within joint project, supported by Huawei Tech. Co., Ltd, China.

\section{REFERENCES}

[1] Cisco, "Cisco visual networking index: Global mobile data traffic forecast update, 2012 - 2017," 2013. [Online]. Available: http://www.cisco.com 
[2] Qualcomm, "LTE heterogeneous networks," 2011. [Online]. Available: http://www.qualcomm.com/media/documents/qualcomm-researchlte-heterogeneous-networks

[3] A. Damnjanovic, J. Montojo, Y. Wei, T. Ji, T. Luo, M. Vajapeyam, T. Yoo, O. Song, and D. Malladi, "A survey on 3GPP heterogeneous networks," IEEE Wireless Communications, vol. 18, no. 3, pp. 10-21, 2011.

[4] J. Rodriguez, P. Marques, A. Radwan, K. Moessner, R. Tafazolli, M. Raspopoulos, S. Stavrou, P. Trapps, D. Noquet, K. Sithamparanathan, A. Gomes, R. Piesiewicz, H. Mokrani, A. Foglar, and C. Verikoukis, "Cognitive radio and cooperative strategies for power saving in multistandard wireless devices," in Future Network and Mobile Summit, 2010, june 2010, pp. $1-8$

[5] D. Feng, C. Jiang, G. Lim, L. Cimini, Jr., G. Feng, and G. Li, "A survey of energy-efficient wireless communications," IEEE Communications Surveys \& Tutorials, to be published, early Access.

[6] C. Yiping and Y. Yuhang, "A new 4G architecture providing multimode terminals always best connected services," Wireless Communications, IEEE, vol. 14 , no. 2 , pp. $36-41$, april 2007.

[7] N. Niebert, A. Schieder, H. Abramowicz, G. Malmgren, J. Sachs, U. Horn, C. Prehofer, and H. Karl, "Ambient networks: an architecture for communication networks beyond 3G," IEEE Wireless Communications, vol. 11, no. 2, pp. 14-22, 2004.

[8] IEEE Standard for Local and Metropolitan Area Networks- Part 21: Media Independent Handover, IEEE Std. 802.21-2008, 2009.

[9] X. Yan, Y. A. ekerciolu, and S. Narayanan, "A survey of vertical handover decision algorithms in fourth generation heterogeneous wireless networks," Computer Networks, vol. 54, no. 11, pp. 1848 - 1863, 2010.

[10] W. Song, W. Zhuang, and Y. Cheng, "Load balancing for cellular/wlan integrated networks," IEEE Network, vol. 21, no. 1, pp. 27-33, 2007.

[11] G. Auer, V. Giannini, C. Desset, I. Godor, P. Skillermark, M. Olsson, M. Imran, D. Sabella, M. Gonzalez, O. Blume, and A. Fehske, "How much energy is needed to run a wireless network?" Wireless Communications, IEEE, vol. 18, no. 5, pp. 40 -49, october 2011.

[12] A. Murabito, "A comparison of efficiency, throughput, and energy requirements of wireless access points," University of New Hampshire, InterOperability Laboratory, WLAN Consortium, Tech. Rep., 2009.

[13] I. Ashraf, F. Boccardi, and L. Ho, "Sleep mode techniques for small cell deployments," Communications Magazine, IEEE, vol. 49, pp. 72-79-, 2011.

[14] 4G++, "Deliverable D4.1, Inter-cell interference coordination," Advanced Performance Boosting Techniques in 4th Generation Wireless Systems, 2012. 